\title{
Qualidade fisiológica e lipídios totais de sementes de pinhão-manso (Jatropha curcas L.) em função de épocas de colheita
}

\author{
Carlos A. D. Ducca ${ }^{1}$, Nátali M. de Souza ${ }^{2}$ \& Cássio E. C. Prete ${ }^{3}$
}

\author{
Palavras-chave: \\ maturidade fisiológica \\ teor de lipídios \\ germinação \\ biocombustíveis
}

\begin{abstract}
R E S U M O
Com a crescente demanda por óleos para a produção de biocombustíveis, espécies que apresentem elevado conteúdo de óleo, como o pinhão-manso (Jatropha curcas L.), são alternativas importantes, frente às opções tradicionais. Tendo em vista que o florescimento se distribui ao longo do ciclo de desenvolvimento da cultura do pinhão-manso objetivou-se, com este trabalho, determinar a época de colheita em dias após o florescimento, adequada para maximização do rendimento de óleo e da qualidade fisiológica de sementes. Em duas safras agrícolas, 2010/2011 (Ano 1) e 2011/2012 (Ano 2) foram colhidos, no município de Paranavaí (PR), frutos de pinhão-manso, em cinco épocas, determinadas por dias após o florescimento: 30, 45, 60, 75 e 90. Em delineamento inteiramente aleatorizado, em esquema de parcelas subdivididas, foram determinados o conteúdo de água, a massa de matéria seca, lipídios totais, emergência em areia e o índice de velocidade de emergência. Pelo comportamento das variáveis as colheitas devem ser realizadas a partir de setenta e cinco dias após o florescimento.
\end{abstract}

\section{Key words:}

physiological maturity

lipids content

germination

biofuels

\section{Physiological quality and total lipids of physic nut seeds (Jatropha curcas L.) according to harvest time}

\begin{abstract}
A B S T R A C T
With the increasing demand for oils for biofuel production, species with high oil content, like physic nut (Jatropha curcas L.), are important alternatives in the face of traditional options. Considering that flowering is distributed throughout the development cycle of physic nut crop, the aim of this work was to determine the harvest time, in days after flowering, appropriate to maximize oil yield and physiological quality of seed. In two growing seasons, 2010/2011 (Year 1) and 2011/2012 (Year 2), at Paranavaí in the State of Paraná. Physic nut fruits were harvested at five times, determined by days after flowering: 30,45 , 60,75 e 90. In completely randomized design, in a split-plot design the water content, dry matter, total lipids, seedling emergence in sand and speed of emergence-index were determined. By the behavior of the variables, harvests should be performed from seventyfive days after flowering.
\end{abstract}

Protocolo 009-2014 - 09/01/2014 • Aprovado em 21/11/2014 • Publicado em 02/03/2015

${ }^{1}$ Instituto Emater. São Carlo do Ivaí, PR. E-mail: carlosducca@uol.com.br

${ }^{2}$ Programa de Pós-graduação em Agronomia/UEL. Londrina, PR. E-mail: natali.maidl@gmail.com (Autora correspondente)

${ }^{3}$ Departamento de Agronomia/UEL. Londrina, PR. E-mail: cassio@uel.br 


\section{INTRODUÇÃo}

De modo geral, as sementes que armazenam óleo se configuram como importantes commodities agrícolas tendo sido destinadas primariamente para fins nutricionais. Tem-se constatado, nos últimos anos, crescimento do uso desses óleos para a produção de combustíveis, fato este justificado em parte pelo rápido aumento nos custos do petróleo, pela preocupação ambiental acerca dos impactos causados e pela utilização de combustíveis fósseis, além da necessidade de desenvolver fontes renováveis a partir de recursos domésticos ou mesmo a partir de materiais crus provenientes das indústrias (Dyer et al., 2008).

De acordo com dados da ANP (2013), cerca de 77,4\% da produção nacional de biodiesel provêm do óleo de soja. No entanto, a cultura da soja apresenta baixo rendimento de óleo, em torno de 150 a $200 \mathrm{~g} \mathrm{~kg}^{-1}$ (Wilson, 2004) se comparada com o pinhão-manso, entre 280 e $370 \mathrm{~g} \mathrm{~kg}^{-1}$ (Arruda et al., 2004), por exemplo.

Além dos altos rendimentos em óleo, o pinhão-manso apresenta características agronômicas de interess, como elevado rendimento de grãos, óleo com qualidade adequada para a produção de biocombustível (Abdelgadir et al., 2008), manejo compatível com o perfil da agricultura familiar (Laviola et al., 2011), adaptação a diferentes regiões climáticas, além de não concorrer diretamente com a produção de alimentos (Freitas et al., 2011). Outro aspecto importante é a qualidade nutricional da torta resultante da extração de óleo, a qual apresenta teores consideráveis de nitrogênio, fósforo e potássio, podendo ser utilizada como biofertilizante (Souza et al., 2009).

O cultivo de pinhão-manso ainda depende, porém, de muita informação técnica para ser viabilizado. Dentre os diversos fatores limitantes à produção em escala pode-se citar o florescimento e a maturação dos frutos desuniformes (Alam et al., 2011; Brasileiro et al., 2012), o que acarreta em necessidade de colheitas escalonadas e reflete em aumento do custo de produção devido à carência de mão de obra constante durante a frutificação. Conforme Marcos Filho (2005), o ponto de maturidade fisiológica de sementes é marcado pelo máximo acúmulo de massa de matéria seca, máxima qualidade fisiológica, além de perda de água. Neste sentido, determinações que avaliem esses atributos podem ser indicadores significativos da maturidade de sementes de pinhão-manso.

O objetivo deste trabalho foi determinar a época de colheita em dias após o florescimento, adequada à maximização do rendimento de óleo para a produção de biocombustível e a qualidade fisiológica de sementes de pinhão-manso.

\section{Material e Métodos}

O experimento foi conduzido entre os anos de 2010 e 2012 em Unidade de Observação (U.O.) de pinhão-manso, do Instituto Paranaense de Assistência Técnica e Extensão Rural (Emater), no município de Paranavaí, estado do Paraná, sob coordenadas geográficas de $23^{\circ} 04^{\prime} 22^{\prime \prime} \mathrm{S}$ e $52^{\circ} 27^{\prime} 54^{\prime \prime} \mathrm{W}$ e 440 $\mathrm{m}$ de altitude. $\mathrm{O}$ clima da região, segundo a classificação de Köppen, é Cfa, ou seja, clima subtropical, com temperatura média no mês mais frio abaixo de $18{ }^{\circ} \mathrm{C}$ (mesotérmico) e temperatura média no mês mais quente acima de $22^{\circ} \mathrm{C}$. Apresenta verões quentes, geadas pouco frequentes e tendência de concentração das chuvas nos meses de verão; contudo, sem estação seca definida (Caviglione et al., 2000). As plantas de pinhão-manso foram introduzidas no local em outubro de 2008. O solo da U.O. é classificado como Latossolo Vermelho Distrófico típico (EMBRAPA, 2013), de textura arenosa, com $830 \mathrm{~g} \mathrm{~kg}^{-1}$ de areia, $34 \mathrm{~g} \mathrm{~kg}^{-1}$ de silte e $136 \mathrm{~g} \mathrm{~kg}^{-1}$ de argila. A análise química de rotina do solo, amostrado de $0-0,20 \mathrm{~m}$, demonstrou: $\mathrm{pH}\left(\mathrm{CaCl}_{2}\right)=4,8 ; \mathrm{H}+\mathrm{Al}^{3+} \mathrm{e} \mathrm{Al}^{3+}=3,18$ e 0,0 $\mathrm{cmol}_{c} \mathrm{dm}^{-3} ; \mathrm{Ca}^{2+}, \mathrm{Mg}^{2+}$ e $\mathrm{K}^{+}=1,82 ; 0,55$ e $0,12 \mathrm{cmol}_{c} \mathrm{dm}^{-3}$; CTC $\left(\mathrm{pH} \mathrm{7,0)}=5,67 \mathrm{mmol} \mathrm{dm}^{-3}\right.$; P (Mehlich-1) $=20,0 \mathrm{mg}$ $\mathrm{dm}^{-3} ; \mathrm{C}$ orgânico total $=10,17 \mathrm{~g} \mathrm{dm}^{-3} ; \mathrm{V}=43,92 \%$. Visando adequações de fertilidade, seguiram-se as recomendações de Laviola \& Dias (2008).

Os frutos de pinhão-manso foram coletados em duas safras agrícolas, sendo a primeira compreendida entre outubro de 2010 e janeiro de 2011 (Ano 1) e a segunda entre outubro de 2011 e janeiro de 2012 (Ano 2). Os tratamentos consistiram em épocas de colheita, em cinco níveis determinados por dias após o florescimento (DAF): 30, 45, 60, 75 e 90 DAF. A primeira época de colheita se caracterizou por frutos totalmente verdes e sementes brancas, a segunda época por frutos verdes e sementes amarronzadas, a terceira por frutos verdes e amarelos com sementes pretas brilhantes, a quarta época por frutos amarelos com manchas marrons e totalmente úmidos com sementes pretas e a última época por frutos secos de marrons a pretos com sementes pretas e opacas.

As variáveis determinadas foram conteúdo de água, massa de matéria seca, lipídios totais, emergência em areia e índice de velocidade de emergência (IVE). O conteúdo de água e a massa de matéria seca foram determinados segundo as Regras para análise de sementes (RAS) (Brasil, 2009), empregando-se três repetições de seis sementes para cada época de colheita. Os lipídios totais foram determinados através do método de Soxhlet, com hidrólise ácida prévia. Foram empregadas amostras de 100 sementes em cada época de colheita, analisadas em triplicata. A hidrólise ácida consistiu em adição de $50 \mathrm{~mL}$ de água destilada e $60 \mathrm{~mL}$ de ácido clorídrico (4 molar) a $5 \mathrm{~g}$ de sementes moídas submetendo a solução a aquecimento a $300^{\circ} \mathrm{C}$ por $30 \mathrm{~min}$. Após filtragem da solução e secagem as amostras foram conduzidas ao extrator de Soxhlet, empregando-se éter de petróleo como solvente e tempo de refluxo de $2 \mathrm{~h} 30 \mathrm{~min}$.

Os testes relacionados à qualidade fisiológica das sementes foram realizados em areia, com sementes depositadas em bandejas a $3 \mathrm{~cm}$ de profundidade e com substrato mantido com $600 \mathrm{~g}_{\text {de }}$ água $\mathrm{kg}^{-1}$ de substrato. A emergência se caracterizou pelo número de plântulas emergidas após quatorze dias. $\mathrm{O}$ índice de velocidade de emergência (IVE) foi determinado de acordo com o método descrito por Maguire (1962) em que: (número de plântulas normais/dias até a primeira contagem) + ... + (número de plântulas normais/dias até a última contagem).

O delineamento experimental foi inteiramente aleatorizado em esquema de parcelas subdivididas devido ao fato de que as coletas foram realizadas em dois anos consecutivos nas mesmas unidades experimentais considerando-se épocas como "parcelas" e "anos" como subparcelas no modelo de análise de variância (Gomes, 2009). As análises estatísticas foram realizadas no software Sisvar (Ferreira, 2011) cujos 
desdobramentos para efeitos de significativos de interação foram realizando através de variâncias complexas e das épocas de colheita por meio de análise de regressão polinomial.

\section{Resultados e Discussão}

Os dados climáticos foram obtidos a partir da estação meteorológica do Instituto Agronômico do Paraná (IAPAR) distante $2000 \mathrm{~m}$ da U.O. Durante o período de desenvolvimento dos frutos esses dados demonstram que as condições de temperatura e precipitação foram favoráveis ao desenvolvimento das plantas (Figura 1A e 1B).

Em termos gerais e apesar da ausência de precipitação entre o período de 11 e 17 de novembro no Ano 2, não foi observado abortamento floral, o que está de acordo com informações de Arruda et al. (2004), que asseguram que o pinhão-manso é uma espécie exigente em insolação porém com forte resistência à seca.

Para as variáveis conteúdo de água $(\mathrm{p}<0,0001)$, emergência em areia $(\mathrm{p}=0,0008)$ e índice de velocidade de emergência ( $\mathrm{p}$ $=0,0331$ ) foram observados efeitos da interação entre épocas de colheita $\mathrm{x}$ ano porém o desdobramento para os efeitos de ano demonstraram não haver ajuste de modelos para estes. Com relação às variáveis massa de matéria seca $(\mathrm{p}<0,0001) \mathrm{e}$ lipídios totais ( $\mathrm{p}<0,0001)$, os efeitos observados foram apenas das épocas de colheita.

O conteúdo de água decresceu linearmente com o avanço das épocas de colheita no tempo (Figura 2A). O trabalho de Santos et al. (2012), que realizaram colheitas em estádios baseados na coloração dos frutos, obteve resultados com tendências semelhantes mas distintos em valores, fato este que também foi observado por Dranski et al. (2010). A terceira época de colheita demonstrada por Santos et al. (2012) apresentou conteúdo de água de aproximadamente $500 \mathrm{~g} \mathrm{~kg}^{-1}$ no entanto, equivale à quarta época deste trabalho (75 DAF) e não à terceira (60 DAF) em que os conteúdos de água se assemelham. Com relação ao trabalho de Dranski et al. (2010), o conteúdo de água variou de aproximadamente 670 a $140 \mathrm{~g} \mathrm{~kg}^{-1}$ em seis estágios de maturação.

Essas diferenças podem ser atribuídas a acessos geneticamente distintos de pinhão-manso visto que não há, no mercado, cultivares disponíveis e, consequentemente, a uniformidade genética se encontra comprometida; sendo assim, a tendência dos comportamentos é mais considerável que os valores absolutos em si.

A.

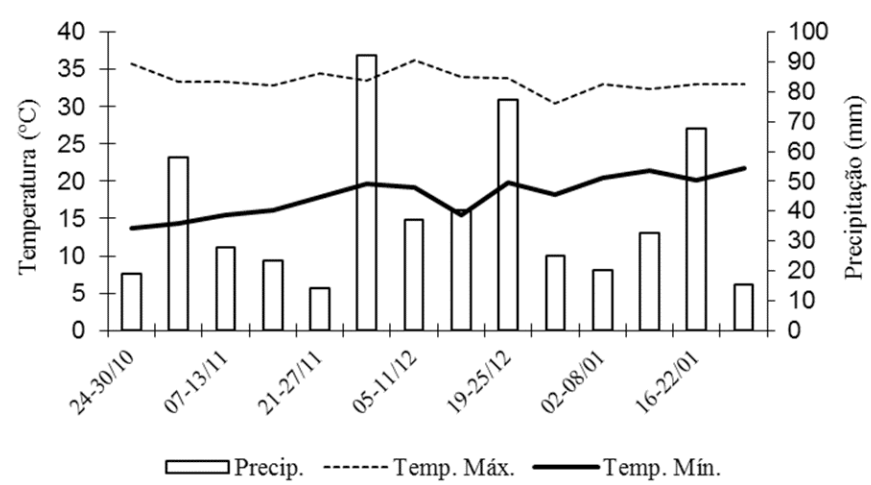

Nos estágios iniciais de desenvolvimento da semente o conteúdo de água elevado é necessário para que o fluxo dos compostos fotossintetizados possa ocorrer da planta-mãe para a semente em formação dando origem às reservas (Corvello et al., 1999). Isto ocorre até que o ponto de máximo acúmulo de matéria seca também

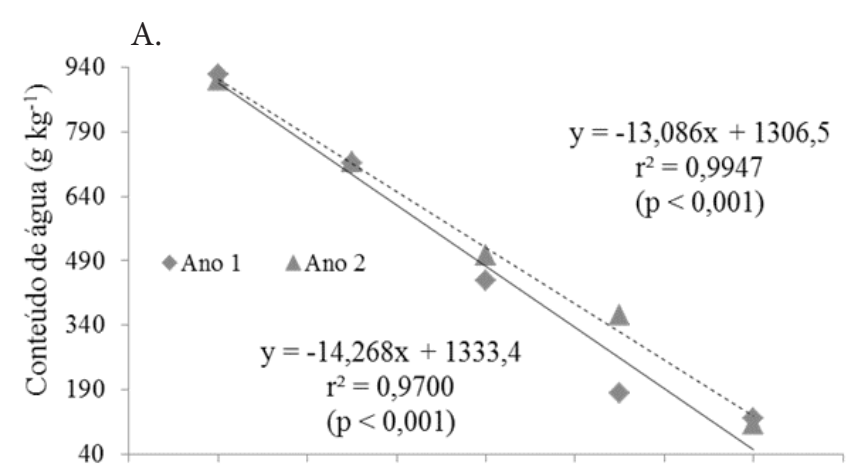

B.

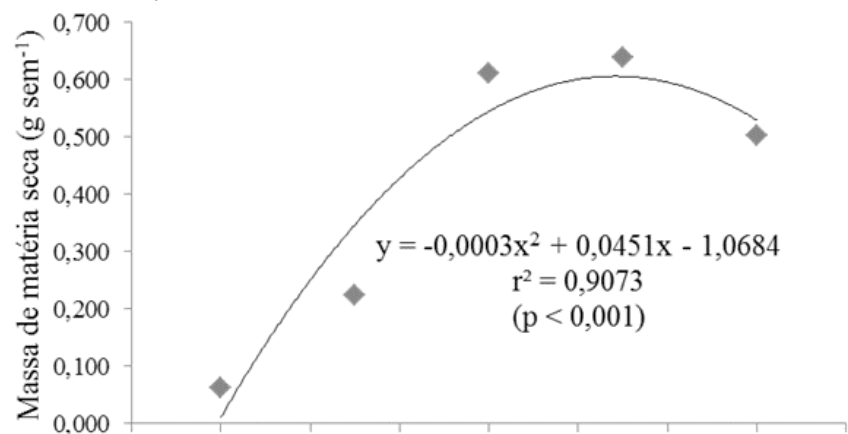

C.

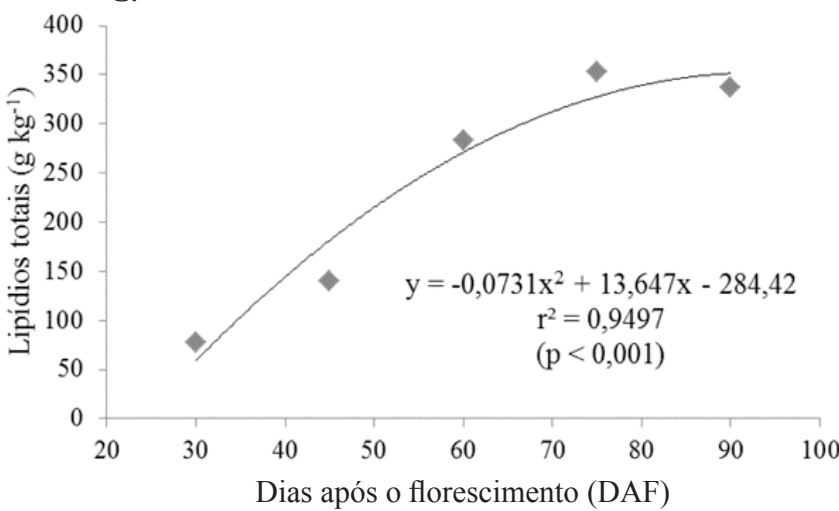

Figura 2. Conteúdo de água (A), massa de matéria seca (B) e lipídios totais $(C)$ das sementes de pinhão-manso em função das épocas de colheita, em dias após o florescimento

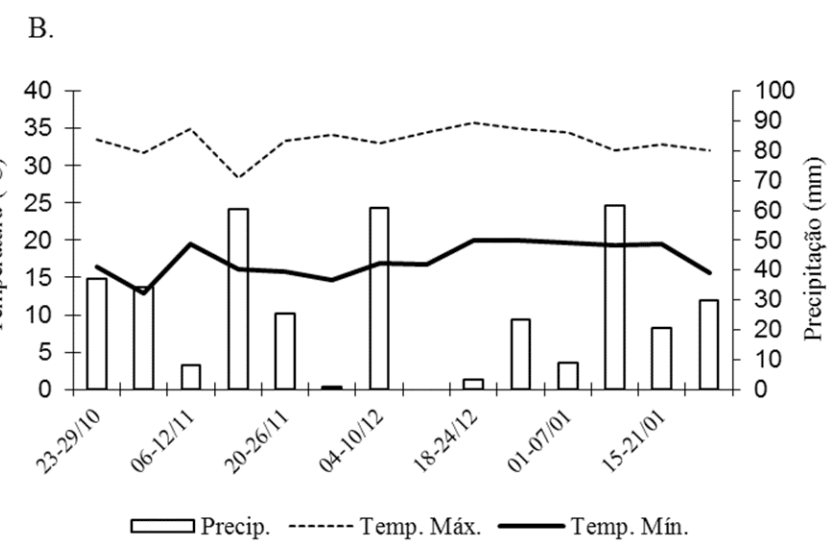

Figura 1. Precipitação, temperaturas máxima e mínima durante o período de condução do ensaio: Ano 1 (A); Ano 2 (B) 
ocorra, levando à redução do conteúdo de água (Marcos Filho, 2005).

Como atributo inerente ao desenvolvimento de sementes a variável massa de matéria seca (Figura $2 \mathrm{~B}$ ) teve ponto de ótimo aos $74 \mathrm{DAF}$, indicando que colheitas realizadas praticamente a partir da quarta época, seriam adequadas. A massa de matéria seca de sementes, associada a outros indicadores, tem sido relatada na literatura como uma boa referência da maturidade fisiológica em diversas espécies, como Caesalpinia echinata (Aguiar et al., 2007), Cedrella fissilis (Corvello et al., 1999), Dalbergia nigra (Martins \& Silva, 1997), Torresia acreana (Firmino et al., 1996), Mimosa caesalpiniifolia (Alves et al., 2005), etc até mesmo para Jatropha curcas (Dranski et al., 2010). No trabalho de Dranski et al. (2010) a massa de matéria seca variou de 220 a $302 \mathrm{mg}$ semente $^{-1}$, enquanto que neste trabalho o valor máximo esteve em aproximadamente $630 \mathrm{mg} \mathrm{semente}^{-1}$.

A variável lipídios totais (Figura $2 \mathrm{C}$ ) apresentou comportamento quadrático semelhante ao da variável massa de matéria seca. Embora o ponto de máximo não corresponda ao tratamento avaliado neste experimento, ao considerar lipídios totais e massa de matéria seca, pode-se afirmar que, a partir e $75 \mathrm{DAF}$, as colheitas seriam adequadas visando à maximização de rendimento de óleo. Levando em consideração que, nesta época, os frutos apresentavam coloração amarela com manchas marrons, informações do trabalho de Teixeira (1987) confirmam a proposição de que esta seja a época adequada para a colheita, pois afirmam que neste estádio as sementes contêm o teor máximo de óleo sendo importante, no entanto, considerar fatores genéticos e ambientais na tomada de decisão.

Como Arruda et al. (2004) descreveram, os teores de lipídios alcançados, em torno de $350 \mathrm{~g} \mathrm{~kg}^{-1}$ aos $75 \mathrm{DAF}$, se encontram em patamares consideráveis e que impulsionam a utilização do pinhão-manso como matéria-prima para a produção de biocombustíveis. Dados de Teixeira (2005) relatam potencial para produção de $1600 \mathrm{~L} \mathrm{ha}^{-1}$. Outras espécies, como a mamona, apesar de apresentarem conteúdos elevados (entre 450 e $500 \mathrm{~g} \mathrm{~kg}^{-1}$ ) apresentam rendimentos por unidade de área que são inferiores aos alcançados pelo pinhão-manso, que estão ao redor de $720 \mathrm{~L} \mathrm{ha}^{-1}$ (Miragaya, 2005).

$\mathrm{O}$ efeito das épocas de colheita sobre a emergência em areia (Figura $3 \mathrm{~A}$ ) foi linear positivo demonstrando que com o avanço das épocas a qualidade das sementes é incrementada.

Os valores para emergência observados a partir da quarta época de colheita, ou seja, frutos com coloração amarela com manchas marrons e sementes pretas, demonstram condições de colheita. Relatos de Dranski et al. (2010) corroboram com essas informações, de maneira que recomendam colheita com valores de emergência superiores a $60 \%$. Para Dagar et al. (2004), há correlação positiva entre o aumento da germinação e a massa fresca da semente, sendo que os maiores valores de germinação são atingidos em sementes com massas superiores a $300 \mathrm{mg}$. Embora os patamares não sejam comparáveis com aqueles estabelecidos pela legislação às grandes culturas, cabe ressaltar que se trata de genótipo não melhorado o que explica, em parte, a expressão desses atributos.

O efeito das épocas de colheita sobre o índice de velocidade de emergência (Figura 3B) foi semelhante à emergência, ou seja, linear positivo. Tal como relatado por Dranski et al. (2010), as sementes originárias das primeiras épocas de colheita não

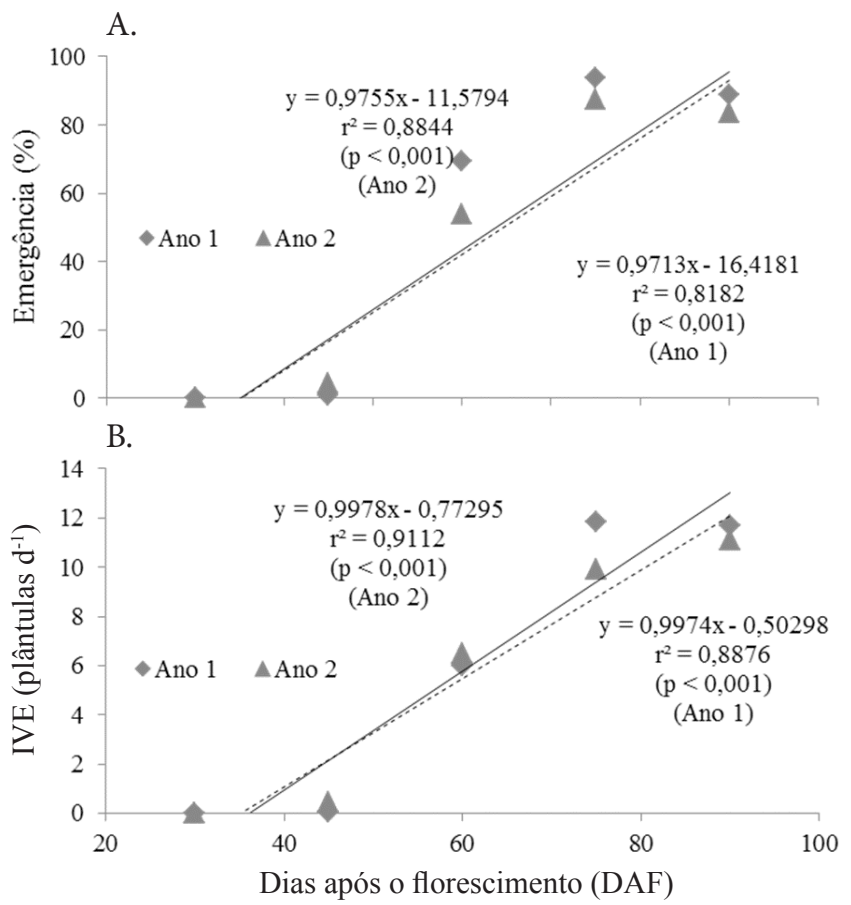

Figura 3. Emergência de plântulas (A) e índice de velocidade de emergência - IVE (B), em função de épocas de colheita, em dias após o florescimento, nos dois anos de ensaio

apresentaram germinação; portanto, o índice de velocidade de emergência também foi nulo nessas épocas porém Dranski et al. (2010) determinaram valores de IVE superiores a 15 enquanto que neste trabalho os valores máximos estiveram próximos a 14. Talvez as diferenças encontradas entre os dois trabalhos estejam na metodologia empregada em que Dranski et al. (2010) realizaram leituras diárias e neste trabalho as leituras foram realizadas em dois momentos.

Tendo em vista que até o presente momento não há cultivares de pinhão-manso disponíveis no mercado, estudar as características de produção dos acessos torna-se ferramenta importante subsidiando os futuros programas de melhoramento.

\section{Conclusão}

As colheitas devem ser realizadas a partir dos setenta e cinco dias após o florescimento visando maximizar os rendimentos de óleo, a massa de matéria seca e a qualidade fisiológica das sementes de pinhão-manso.

\section{Agradecimentos}

À Coordenação de Aperfeiçoamento de Pessoal de Nível Superior (CAPES) pelo auxílio financeiro através de bolsa de pós-graduação e ao Instituto EMATER/PR, pela concessão da área experimental.

\section{Literatura Citada}

Abdelgadir, H. A.; Johnson, S. D.; Staden, J. van. Approaches to improve seed production of Jatropha curcas L. South African Journal of Botany, v.74, p.359, 2008. http://dx.doi.org/10.1016/j. sajb.2008.01.023 
Aguiar, F. F. A.; Pinto, M. M.; Tavares, A. R.; Kanashiro, S. Maturação de frutos de Caesalpinia echinata Lam. Pau-Brasil. Revista Árvore, v.31, p.1-6, 2007. http://dx.doi.org/10.1590/S010067622007000100001

Alam, N. C. N.; Abdullah, T. L.; Abdullah, N. A. Flowering and fruit set under malaysian climate of Jatropha curcas L. American Journal of Agricultural and Biological Sciences, v.6, p.142-147, 2011. http:// dx.doi.org/10.3844/ajabssp.2011.142.147

Alves, E. U.; Sader, R.; Bruno, R. L. de A.; Alves, A.U. Maturação fisiológica de sementes de Sabiá. Revista Brasileira de Sementes, v.27, p.1-8, 2005. http://dx.doi.org/10.1590/S010131222005000100001

ANP - Agência Nacional do Petróleo, Gás Naturale Biocombustíveis. Anuário estatístico brasileiro do petróleo, gás natural e biocombustíveis 2013. Rio de Janeiro: ANP, 2013. 232p.

Arruda, F. P.; Beltrão, N. E. de M.; Andrade, A. P.; Pereira, W. E.; Severino, L. S. Cultivo de pinhão-manso (Jatropha curcas L.) como alternativa para o semi-árido nordestino. Revista Brasileira de Oleaginosas e Fibrosas, v.8, p.789-799, 2004.

Brasil. Ministério da Agricultura, Pecuária e Abastecimento. Regras para análise de sementes. Brasília: Mapa/ACS, 2009. 399p.

Brasileiro, B. G.; Dias, D. C. F.; Bhering, M. C.; Dias, L. A. S. Floral biology characterization of seed germination in physic nut (Jatropha curcas L.) Revista Brasileira de Sementes, v.34, p.556562, 2012. http://dx.doi.org/10.1590/S0101-31222012000400005

Caviglione, J. H.; Kiihl, L. R. B.; Caramori, P. H.; Oliveira, D. Cartas climáticas do Paraná. Londrina: IAPAR, 2000. <http://www. iapar.br/modules/conteudo/conteudo.php?conteudo=677 $>.14$ Jun. 2013.

Corvello, W. B. V.; Villela, F. A.; Nedel, J. L.; Peske, S.T. Maturação fisiológica de sementes de cedro (Cedrela fissilis Vell.). Revista Brasileira de Sementes, v.21, p.23-27, 1999.

Dagar, J. C.; Bhagwan, H.; Kumar, Y. Seed germination studies of Salvadora persica and Jatropha curcas. Indian Journal of Forestry, v.27, p.283-289, 2004.

Dranski, J. A. L.; PintoJúnior, A. S.; Steiner, F.; Zoz, T.; Malavasi, U. C.; Malavasi, M. M.; Guimarães, V. F. Physiological maturity of seeds and colorimetry of fruits of Jatropha curcas L. Revista Brasileira de Sementes, v.32, p.158-165, 2010. http://dx.doi.org/10.1590/ S0101-31222010000400018

Dyer, J. M.; Stymne, S.; Green, A. G.; Carlsson, S. High-value oils from plants. The Plant Journal, v.54, p.640-655, 2008. http://dx.doi. org/10.1111/j.1365-313X.2008.03430.x

EMBRAPA - Empresa Brasileirade Pesquisa Agropecuária. Sistema Brasileiro de Classificação de Solos. 3.ed. Brasília: EMBRAPA, 2013. 353p.

Ferreira, D. F. Sisvar: a computer statistical analysis system. Ciência e Agrotecnologia, v. 35, p. 1039-1042, 2011.
Firmino, J. L.; Santos, D. S. B.; Santos Filho, B. G. Características físicas e fisiológicas de sementes de cerejeira (Torresia acreana Ducke) quando as sementes foram coletadas do chão ou do interior dos frutos. Revista Brasileira de Sementes, v.18, p.2832, 1996.

Freitas, R. G.; Missio, R. F.; Matos, F. S.; Resende, M. D. V.; Dias, L. A. S. Genetic evaluation of Jatropha curcas: An important oil seed for biodiesel production. Genetics and Molecular Research, v.10, p.1490-1498, 2011. http://dx.doi.org/10.4238/vol10-3gmr1146

Gomes, F. P. Curso de estatística experimental. 15.ed. Piracicaba: FEALQ, 2009. 451p.

Laviola, B. G.; Bhering, L. L.; Mendonça, S.; Rosado, T. B.; Albrecht, J. C. Caracterização morfo-agronômica do banco de germoplasma de pinhão-manso na fase jovem. Bioscience Journal, v.27, p.371379, 2011.

Laviola, B. G.; Dias, L. A. S. Teor e acúmulo de nutrientes em folhas e frutos de pinhão-manso. Revista Brasileira de Ciência do Solo, v.32, p.1969-1975, 2008. http://dx.doi.org/10.1590/S010006832008000500018

Maguire, J. D. Speed germination - aid in selection and evaluation for seedling emergence and vigor. Crop Science, v.2, p.176-177, 1962. http://dx.doi.org/10.2135/cropsci1962.0011183X000200020033x

Marcos Filho, J. Fisiologia de sementes de plantas cultivadas. Piracicaba: FEALQ, 2005. 495p.

Martins, S. V.; Silva, D. D. Maturação e época de colheita de sementes de Dalbergia nigra (Vell.) Fr. All. exBenth. Revista Brasileira de Sementes, v.19, p.96-99, 1997.

Miragaya, J. C. G. Biodiesel: Tendências no mundo e no Brasil. Informe Agropecuário, v.26, p.7-13, 2005.

Santos, S. B.; Martins, M. A.; Aguilar, P. R. M.; Caneschi, A. L.; Carneiro, A. C. O.; Dias, L. A. S. Acúmulo de matéria seca e óleo nas sementes de pinhão-manso e qualidade de óleo extraído. Revista Brasileira de Engenharia Agrícola e Ambiental, v.16, p.209-215, 2012. http://dx.doi.org/10.1590/S141543662012000200012

Souza, A. D. V.; Fávaro, S. P.; Ítavo, L. C. V.; Roscoe, R. Caracterização química de sementes e tortas de pinhão-manso, nabo-forrageiro e crambe. Pesquisa Agropecuária Brasileira, v.44, p.1328-1335, 2009. http://dx.doi.org/10.1590/S0100-204X2009001000017

Teixeira, J. P. F. Teor e composição do óleo de sementes de Jatropha sp. Bragantia, v.46, p.151-157, 1987. http://dx.doi.org/10.1590/ S0006-87051987000100017

Teixeira, L. C. Potencialidades de oleaginosas para produção de biodiesel. Informe Agropecuário, v.26, p.18-27, 2005.

Wilson, R. F. Seed composition. In: Boerma, H. R.; Specht, J. E. (ed.). Soybeans: Improvement, production, and uses. 3.ed. Madison: American Society of Agronomy, Crop Science Society of America, Soil Science Society of America, 2004. cap.13, p.621-677. 